\title{
Expression profile analysis based on DNA microarray for patients undergoing off-pump coronary artery bypass surgery
}

\author{
YUNPENG SUN ${ }^{1}$, YONGSHENG GAO ${ }^{1}$, JINGNAN SUN ${ }^{2}$, XUGUANG LIU ${ }^{1}$, \\ DASHI MA $^{1}$, CHUNYE MA ${ }^{1}$ and YONG WANG ${ }^{1}$ \\ ${ }^{1}$ Department of Cardiac Surgery; ${ }^{2}$ Cancer Center, The First Hospital of Jilin University, Changchun, Jilin 130021, P.R. China
}

Received October 27, 2014; Accepted December 3, 2015

DOI: $10.3892 /$ etm.2016.3003

\begin{abstract}
Off-pump coronary artery bypass (OPCAB) surgery is the most effective treatment for coronary heart disease. The aim of this study was to explore the effects of OPCAB on the basis of the associated molecular mechanisms. GSE12486 expression profiles downloaded from the Gene Expression Omnibus database (GEO) were analyzed to identify the differentially expressed genes (DEGs). Principal component analysis (PCA) was conducted based on the expression profiles of DEGs. Function and pathway enrichment of upregulated DEGs was performed, followed by protein-protein interaction (PPI) network construction. Gene Set Enrichment Analysis (GSEA) was used for miRNA enrichment analysis based on expression profiles and prediction of their association with the disease. Cytoscape was applied to construct miRNA regulatory networks of DEGs. In total 64 DEGs were identified, including 63 upregulated and 1 downregulated gene. The first principal component in the PCA analysis was able to distinguish between pre- and post-OPCAB samples. Upregulated DEGs mainly enriched 20 Gene Ontology terms, such as chemokine activity, and 5 pathways including the chemokine signaling pathway. The constructed PPI network contained 234 edges and 55 nodes, and 10 upregulated hub nodes, including FBJ murine osteosarcoma viral oncogene homolog (FOS), were screened. A total of 36 miRNAs, including MIR-224 and MIR-7, were screened by GSEA enrichment analysis. A miRNA regulatory network including 176 edges and 97 nodes was constructed, showing the regulatory relationships between miRNAs and DEGs. For example, early growth response 2 (EGR2) was regulated by 8 miRNAs including MIR-150, MIR-142-3P, MIR-367 and MIR-224. The identified DEGs might play important roles in patients pre- and post-OPCAB surgery via the regulation of associated genes.
\end{abstract}

Correspondence to: Dr Yongsheng Gao, Department of Cardiac Surgery, The First Hospital of Jilin University, 71 Xinmin Street, Changchun, Jilin 130021, P.R. China

E-mail: yongshenggaoo@163.com

Key words: off-pump coronary artery bypass, pathway analysis, protein-protein interaction network, miRNA regulatory network

\section{Introduction}

Coronary artery disease is a defect with a narrowing of the coronary arteries that supply blood and oxygen to the heart (1). Off-pump coronary artery bypass (OPCAB) surgery, in which veins or arteries are grafted to the coronary arteries to bypass atherosclerotic narrowing and improve the blood supply to the heart muscle, reduces the risk of mortality from coronary artery disease (2). However, coronary patients who have undergone OPCAB grafting often suffer from disorders of coagulation and hemostasis (3). Therefore, it is necessary to research the molecular mechanisms underlying the effect of OPCAB.

Expression profile analyses have been used to examine the myocardial stress response to cardiac surgery (4-6). In addition, molecular biology studies have confirmed that several genes, including interleukin 6 (IL6), IL8 and activating transcription factor 3 (ATF3), and certain biological processes are associated with the effect of OPCAB grafting. IL6 and IL8 proteins, released by ischemic cardiac myocytes, are induced following acute ischemia (5,7). Moreover, Tomic et al found that the expression of IL6 tended to increase after OPCAB grafting (8), and Ghorbel et al showed that ventricular heart cells may undergo alterations in chemokine and plasma cytokine levels following OPCAB surgery (9). Furthermore, several important miRNA regulatory relationships in OPCAB samples have been investigated. For example, microRNA (MIR)-494 targets the 3' untranslated region of ATF3 reporter and decreases ATF3 mRNA expression $(10,11)$.

The identification of sensitive genes and miRNAs has become the focus for establishing suitable therapeutic strategies, and gene expression profiling techniques have been widely used to analyze the effects of OPCAB. A number of studies have reported some specific gene expression changes in samples following OPCAB surgery $(9,12,13)$, while a variety of genes have been studied in the fundamental research of OPCAB. Studies have screened for genes associated with the levels of chemokines and cytokine following OPCAB surgery, but little attention has been paid to the corresponding miRNA transcripts (8). In addition, the molecular mechanisms of complications, including hemostasis and coagulation disorders, caused by OPCAB grafting are unclear (14).

In the present study, the data in the gene expression series record GSE12486 were downloaded, to screen for 
differentially expressed genes (DEGs) between samples obtained pre- and post-OPCAB surgery, followed by function and pathway enrichment analysis, as well as protein-protein interaction (PPI) network construction. Based on expression profiles obtained by microarray analysis, Gene Set Enrichment Analysis (GSEA) was used to enrich the miRNAs predicted to have a correlation with OPCAB grafting. Finally, miRNA regulatory networks were constructed to investigate the association between miRNA and DEGs.

\section{Materials and methods}

Microarray data. The gene expression profile GSE12486 was obtained from the Gene Expression Omnibus database (GEO; http://www.ncbi.nlm.nih.gov/geo/) (9). The platform was GPL570 Affymetrix Human Genome U133 Plus 2.0 Array (Affymetrix, Inc., Santa Clara, CA, USA). A total of 10 myocardial samples obtained prior to and after grafting were available; they were collected from 5 patients undergoing OPCAB grafting with cardiopulmonary bypass and cardiac arrest. The pre-surgery samples contained GSM313629, GSM313631, GSM313633, GSM313635 and GSM313637, while post-surgery samples included GSM313638, GSM313639, GSM313640, GSM313641 and GSM313642.

Data preprocessing and DEG screening. The original data were read by the Affymetrix package with R-based software and processed by the Robust Multi-array Analysis (RMA) method (15). The processed data then underwent normalization with a universal background in order to evaluate the expression values. The limma multiple linear regression package (16) was applied for the calculation and analysis of DEGs, with further rectification by the Bayes method (17). The genes with $\mathrm{P}<0.01$ and $\mid \log$ (fold change) $\mid>1$ were selected as DEGs.

Principal component analysis (PCA). The PCA was conducted on the basis of the expression profile of DEGs. PCA is a multivariate statistical analysis that uses linear transformation to convert multiple correlated variables into a set of important linearly uncorrelated variables (18).

Pathway enrichment analysis. Database for Annotation, Visualization and Integrated Discovery (DAVID) is a web-based tool for extracting the biological meaning of large numbers of genes (19). It was used to carry out Gene Ontology (GO) function and Kyoto Encyclopedia of Genes and Genomes (KEGG) pathway enrichment analysis of upregulated DEGs. Based on the principle of hypergeometric distribution, GO and KEGG terms were enriched with the threshold of $\mathrm{P}<0.01$.

Protein-protein interaction (PPI) network construction. A PPI network of DEGs was constructed using the Search Tool for Retrieval of Interacting Genes (STRING) database, which provides integrated knowledge of the known and predicted associations for protein networks (20). The PPI network comprised 'nodes' and 'edges'; each protein was represented by a node, while the interaction of pairwise proteins was shown by an edge. The 5 nodes with the greatest number of connections (high degree) were further screened as the 'hub' genes.
Table I. Top 10 differentially expressed genes (DEGs).

\begin{tabular}{llc}
\hline DEG & Log2FC & P-value \\
\hline FOS & 3.855915 & 0.000293 \\
CCL3 & 3.369698 & $1.05 \mathrm{E}-07$ \\
CCL3L1 & 3.369698 & $1.05 \mathrm{E}-07$ \\
CCL3L3 & 3.369698 & $1.05 \mathrm{E}-07$ \\
EGR2 & 3.149878 & $9.33 \mathrm{E}-06$ \\
NR4A2 & 3.015238 & $3.16 \mathrm{E}-05$ \\
ZFP36 & 2.983598 & $2.55 \mathrm{E}-06$ \\
FOSB & 2.974505 & 0.000304 \\
EGR3 & 2.961837 & $4.57 \mathrm{E}-05$ \\
CH25H & 2.734594 & 0.000209 \\
\hline
\end{tabular}

$\log 2 \mathrm{FC}$ is the logarithmic value of the DEG variation range. FOS, FBJ murine osteosarcoma viral oncogene homolog; CCL3, chemokine (C-C motif) ligand 3; CCL3L1, chemokine (C-C motif) ligand 3-like 1; CCL3L3, chemokine (C-C motif) ligand 3-like 3; EGR2, early growth response 2 ; NR4A2, nuclear receptor subfamily 4 , group A, member 2; ZFP36, ZFP36 ring finger protein; FOSB, FBJ murine osteosarcoma viral oncogene homolog B; EGR3, early growth response 3; $\mathrm{CH} 25 \mathrm{H}$, cholesterol 25-hydroxylase.

miRNA prediction analysis. GSEA is a method for the enrichment analysis of a gene set based on whole genome expression profiling (21). On the basis of the expression profiles obtained by microarray analysis, GSEA was used to enrich the disease-related miRNAs. The related miRNAs were screened with a threshold of false discovery rate (FDR) of $<0.05$.

miRNA regulatory networks. Cytoscape is an open software platform used for visualizing complex networks and integrating these networks with any type of attribute data (22). It was applied for building the regulatory networks of DEGs that were regulated by miRNA.

\section{Results}

Data standardization and DEG screening. After processing by the RMA method, the expression data presented good standardization in the samples taken pre- and post-surgery (Fig. 1). On the basis of the differential expression analysis for GSE12486, a total of 64 DEGs with $\log 2 \mathrm{FCl}>1$ and $\mathrm{P}<0.01$ were obtained, including 63 upregulated genes and 1 downregulated gene. Perilipin 1 (PLIN1) was the only downregulated gene. The top 10 DEGs are presented in Table I.

PCA. The DEGs were processed by the PCA method. GSM313629 and GSM313640 samples were the first principal components pre- and post grafting, respectively. As shown in Fig. 2, the first two principal components (score, 84.88\%) were well able to distinguish between the samples taken pre- and post-surgery.

GO and KEGG pathway enrichment analysis. GO enrichment analysis of upregulated and downregulated DEGs was performed using the DAVID tool. In total, 20 GO terms were 

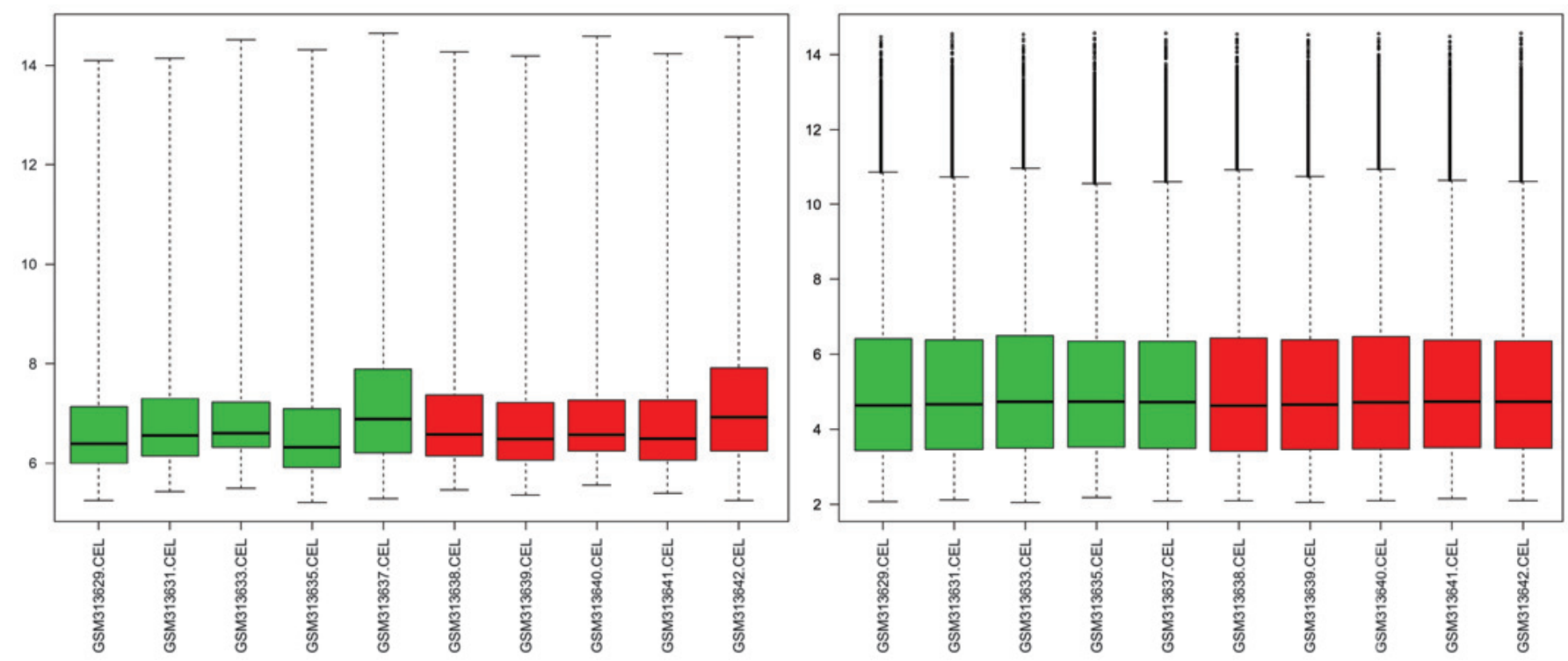

Figure 1. Results of data standardization. The left and right figures are box-plots of pre- and post-standardization data, respectively. The green and red boxes represent pre- and post-surgery samples, respectively.

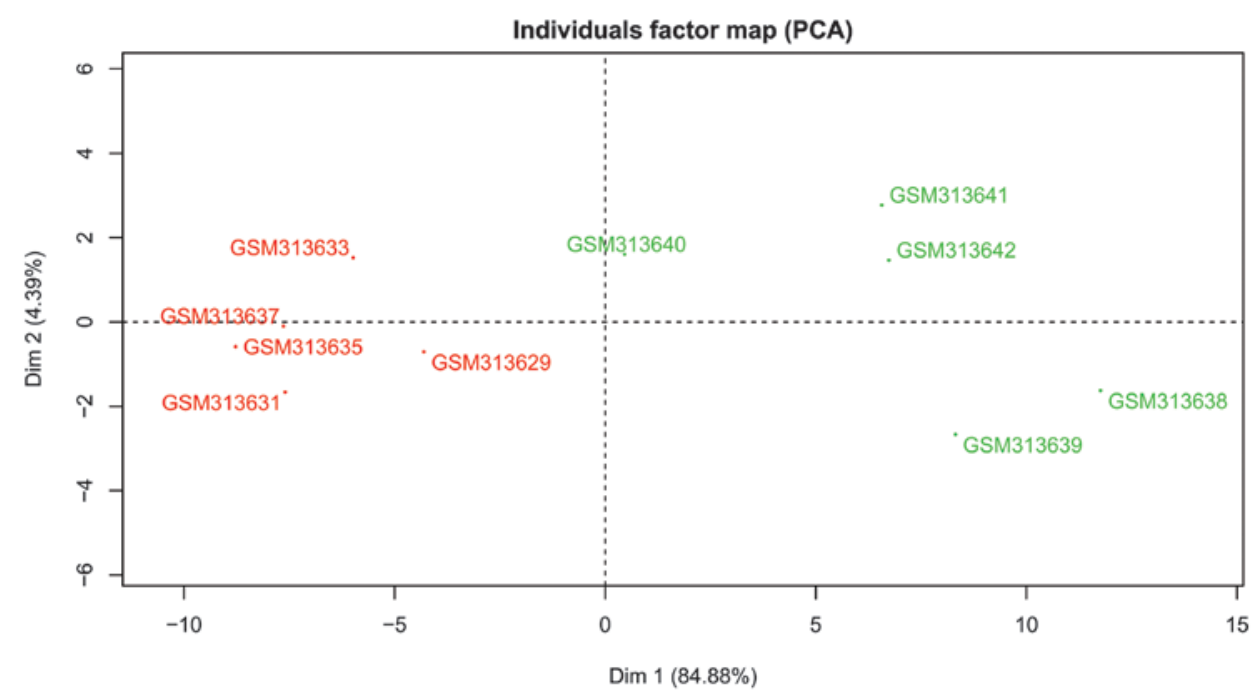

Figure 2. Results of principal component analysis (PCA). The red and green text represent samples of pre- and post-off pump coronary artery bypass surgery, respectively. Dim, dimension.

found to be enriched by the upregulated DEGs (Table II), of which several important functions were associated with immune function, such as inflammatory response, defense response, response to wounding and chemokine receptor binding. IL6, IL8, chemokine (C-C motif) ligand 4 (CCL4), CCL3, CCL2, chemokine (C-X-C motif) ligand 2 (CXCL2) and nuclear factor of $\kappa$ light polypeptide gene enhancer in B-cells inhibitor, $\zeta$ (NFKBIZ) were among the DEGs enriched in these significant functions.

The DAVID tool was also used for KEGG pathway analysis of the screened upregulated DEGs. Only 5 pathways were enriched (Table III), including the chemokine signaling pathway $(\mathrm{P}=2.23 \mathrm{E}-05)$, cytokine-cytokine receptor interaction $(\mathrm{P}=2.24 \mathrm{E}-05)$, Toll-like receptor signaling pathway $(\mathrm{P}=1.08 \mathrm{E}-04)$, NOD-like receptor signaling pathway $(\mathrm{P}=2.09 \mathrm{E}-04)$ and mitogen-activated protein kinase (MAPK) signaling pathway $(\mathrm{P}=0.008586)$. The most significant pathway was the chemokine signaling pathway, which was enriched by the following upregulated DEGs: CCL3, CCL2, IL8, CXCR4, CCL3L1, CXCL2, CCL3L3, CCL8 and CCL4.

PPI network construction. The PPI network, which contained 55 nodes and 234 edges, was constructed on the basis of the STRING database analysis (Fig. 3). Based on the degree (connectivity) of the nodes, the top 10 upregulated hub nodes with higher degrees were screened. These hub genes included FBJ murine osteosarcoma viral oncogene homolog (FOS), jun proto-oncogene (JUN), ATF3, IL6, FBJ murine osteosarcoma viral oncogene homolog B (FOSB), jun B proto-oncogene (JUNB), prostaglandin-endoperoxide synthase 2 (PTGS2), early growth response 1 (EGR1), early growth response 2 (EGR2) and nuclear receptor subfamily 4, group A, member 2 (NR4A2). Among these, FOS showed the highest node degree, which was 33 (Table IV). 
Table II. Functional enrichment analysis for upregulated DEGs.

\begin{tabular}{|c|c|c|c|c|}
\hline Category & GO id & Description & P-value ${ }^{a}$ & Genes \\
\hline BP & GO:0007610 & Behavior & $1.57 \mathrm{E}-15$ & $\begin{array}{l}\text { EGR1, IL6, CCL3, EGR2, CCL2, C5AR1, IL8, PTGS2, } \\
\text { S100A9, CXCL2, NR4A2, CCL8, FOSB, NR4A3, CCL4, } \\
\text { PROK2, FOS, CXCR4, CCL3L1, JUN, CCL3L3, CYR61 }\end{array}$ \\
\hline BP & GO:0042330 & Taxis & $2.21 \mathrm{E}-12$ & $\begin{array}{l}\text { CCL3, IL6, CCL2, C5AR1, IL8, CXCL2, S100A9, CCL8, } \\
\text { CCL4, PROK2, CXCR4, CCL3L1, CCL3L3, CYR61 }\end{array}$ \\
\hline $\mathrm{BP}$ & GO:0006935 & Chemotaxis & $2.21 \mathrm{E}-12$ & $\begin{array}{l}\text { CCL3, IL6, CCL2, C5AR1, IL8, CXCL2, S100A9, CCL8, } \\
\text { CCL4, PROK2, CXCR4, CCL3L1, CCL3L3, CYR61 }\end{array}$ \\
\hline BP & GO:0006954 & $\begin{array}{l}\text { Inflammatory } \\
\text { response }\end{array}$ & $3.29 \mathrm{E}-12$ & $\begin{array}{l}\text { NFKBIZ, IL6, CCL3, CCL2, IL8, S100A8, CXCL2, } \\
\text { S100A9, CCL8, CCL4, S100A12, FOS, PROK2, CXCR4, } \\
\text { CCL3L1, CCL3L3, SELE }\end{array}$ \\
\hline $\mathrm{BP}$ & GO:0007626 & $\begin{array}{l}\text { Locomotory } \\
\text { behavior }\end{array}$ & $8.03 \mathrm{E}-11$ & $\begin{array}{l}\text { CCL3, IL6, CCL2, C5AR1, IL8, CXCL2, S100A9, } \\
\text { NR4A2, CCL8, CCL4, PROK2, CXCR4, CCL3L1, } \\
\text { CCL3L3, CYR61 }\end{array}$ \\
\hline BP & GO:0006952 & $\begin{array}{l}\text { Defense } \\
\text { response }\end{array}$ & $3.10 \mathrm{E}-10$ & $\begin{array}{l}\text { NFKBIZ, IL6, CCL3, CCL2, C5AR1, IL8, S100A8, } \\
\text { CXCL2, S100A9, CCL8, CCL4, S100A12, CD83, } \\
\text { PROK2, FOS, CXCR4, CCL3L1, CCL3L3, SELE }\end{array}$ \\
\hline $\mathrm{BP}$ & GO:0009611 & $\begin{array}{l}\text { Response } \\
\text { to wounding }\end{array}$ & $3.11 \mathrm{E}-09$ & $\begin{array}{l}\text { NFKBIZ, IL6, CCL3, CCL2, IL8, S100A8, CXCL2, } \\
\text { S100A9, CCL8, CCL4, S100A12, FOS, PROK2, CXCR4, } \\
\text { CCL3L1, CCL3L3, SELE }\end{array}$ \\
\hline $\mathrm{BP}$ & GO:0010033 & $\begin{array}{l}\text { Response to } \\
\text { organic substance }\end{array}$ & $3.51 \mathrm{E}-09$ & $\begin{array}{l}\text { EGR1, IL6, EGR2, CCL2, PTGS2, SOCS3, NR4A2, } \\
\text { NR4A3, PMAIP1, JUNB, CD83, FOS, DUSP1, BTG2, } \\
\text { JUN, SELE, MYC, CYR61 }\end{array}$ \\
\hline BP & GO:0045944 & $\begin{array}{l}\text { Positive regulation of } \\
\text { transcription from } \\
\text { RNA polymerase II } \\
\text { promoter }\end{array}$ & 3.39E-07 & $\begin{array}{l}\text { EGR1, FOS, IL6, EGR2, JUN, CSRNP1, NR4A2, ABRA, } \\
\text { NR4A3, MYC, KLF4, JUNB }\end{array}$ \\
\hline $\mathrm{BP}$ & GO:0002237 & $\begin{array}{l}\text { Response to molecule } \\
\text { of bacterial origin }\end{array}$ & $1.55 \mathrm{E}-06$ & FOS, IL6, CCL2, PTGS2, SOCS3, JUN, SELE \\
\hline MF & GO:0008009 & Chemokine activity & $2.25 \mathrm{E}-08$ & CCL3, CCL2, IL8, CCL3L1, CXCL2, CCL3L3, CCL8, CCL4 \\
\hline MF & GO:0042379 & $\begin{array}{l}\text { Chemokine } \\
\text { receptor binding }\end{array}$ & 3.34E-08 & CCL3, CCL2, IL8, CCL3L1, CXCL2, CCL3L3, CCL8, CCL4 \\
\hline MF & GO:0003700 & $\begin{array}{l}\text { Transcription } \\
\text { factor activity }\end{array}$ & $2.99 \mathrm{E}-06$ & $\begin{array}{l}\text { EGR1, MAFF, EGR3, EGR2, CEBPD, NR4A2, NR4A3, } \\
\text { FOSB, JUNB, FOS, ATF3, JUN, CSRNP1, BHLHE40, } \\
\text { MYC, KLF4 }\end{array}$ \\
\hline MF & GO:0005125 & Cytokine activity & $1.02 \mathrm{E}-05$ & $\begin{array}{l}\text { CCL3, IL6, CCL2, IL8, CCL3L1, CXCL2, CCL3L3, } \\
\text { CCL8, CCL4 }\end{array}$ \\
\hline MF & GO:0043565 & $\begin{array}{l}\text { Sequence-specific } \\
\text { DNA binding }\end{array}$ & $1.84 \mathrm{E}-05$ & $\begin{array}{l}\text { EGR1, MAFF, FOS, ATF3, CEBPD, JUN, NR4A2, } \\
\text { NR4A3, FOSB, MYC, KLF4, JUNB }\end{array}$ \\
\hline MF & GO:0030528 & $\begin{array}{l}\text { Transcription } \\
\text { regulator activity }\end{array}$ & $3.70 \mathrm{E}-05$ & $\begin{array}{l}\text { EGR1, MAFF, EGR3, EGR2, CEBPD, NR4A2, NR4A3, } \\
\text { FOSB, JUNB, FOS, ATF3, JUN, CSRNP1, ABRA, } \\
\text { BHLHE40, SIK1, MYC, KLF4 }\end{array}$ \\
\hline MF & GO:0003690 & $\begin{array}{l}\text { Double-stranded } \\
\text { DNA binding }\end{array}$ & 3.76E-05 & EGR1, FOS, JUN, MYC, KLF4, JUNB \\
\hline MF & GO:0043566 & $\begin{array}{l}\text { Structure-specific } \\
\text { DNA binding }\end{array}$ & $2.52 \mathrm{E}-04$ & EGR1, FOS, JUN, MYC, KLF4, JUNB \\
\hline MF & GO:0003677 & DNA binding & 0.002359 & $\begin{array}{l}\text { ZFP36, EGR1, MAFF, EGR3, EGR2, CEBPD, NR4A2, } \\
\text { NR4A3, FOSB, ZNF331, JUNB, FOS, ATF3, JUN, } \\
\text { CSRNP1, BHLHE40, SOX17, MYC, KLF4 }\end{array}$ \\
\hline MF & GO:0008201 & Heparin binding & 0.007664 & CCL2, CCL8, ADAMTS1, CYR61 \\
\hline
\end{tabular}

${ }^{\mathrm{a}}$ Enriched P-value based on the principle of hypergeometric distribution. DEGs, differently expressed genes; GO id, gene ontology identifier; $\mathrm{BP}$, biological process; MF, molecular function. 
Table III. Results of KEGG pathway enrichment analysis for upregulated DEGs.

\begin{tabular}{|c|c|c|c|}
\hline Term & Description & P-value & Genes \\
\hline hsa04062 & $\begin{array}{l}\text { Chemokine } \\
\text { signaling pathway }\end{array}$ & $2.23 \mathrm{E}-05$ & $\begin{array}{l}\text { CCL3, CCL2, IL8, CXCR4, CCL3L1, CXCL2, CCL3L3, CCL8, } \\
\text { CCL4 }\end{array}$ \\
\hline hsa04060 & $\begin{array}{l}\text { Cytokine-cytokine } \\
\text { receptor interaction }\end{array}$ & $2.24 \mathrm{E}-05$ & $\begin{array}{l}\text { CCL3, IL6, CCL2, IL8, CXCR4, CCL3L1, CXCL2, CCL3L3, } \\
\text { CCL8, CCL4 }\end{array}$ \\
\hline hsa04620 & $\begin{array}{l}\text { Toll-like receptor } \\
\text { signaling pathway }\end{array}$ & $1.08 \mathrm{E}-04$ & FOS, CCL3, IL6, IL8, JUN, CCL4 \\
\hline hsa04621 & $\begin{array}{l}\text { NOD-like receptor } \\
\text { signaling pathway }\end{array}$ & $2.09 \mathrm{E}-04$ & IL6, CCL2, IL8, CXCL2, CCL8 \\
\hline hsa04010 & $\begin{array}{l}\text { MAPK signaling } \\
\text { pathway }\end{array}$ & 0.008586 & DUSP5, FOS, DUSP1, JUN, GADD45B, MYC \\
\hline
\end{tabular}

KEGG, Kyoto Encyclopedia of Genes and Genomes; DEGs, differentially expressed genes; NOD, nucleotide-binding oligomerization domain; MAPK, mitogen-activated protein kinase.

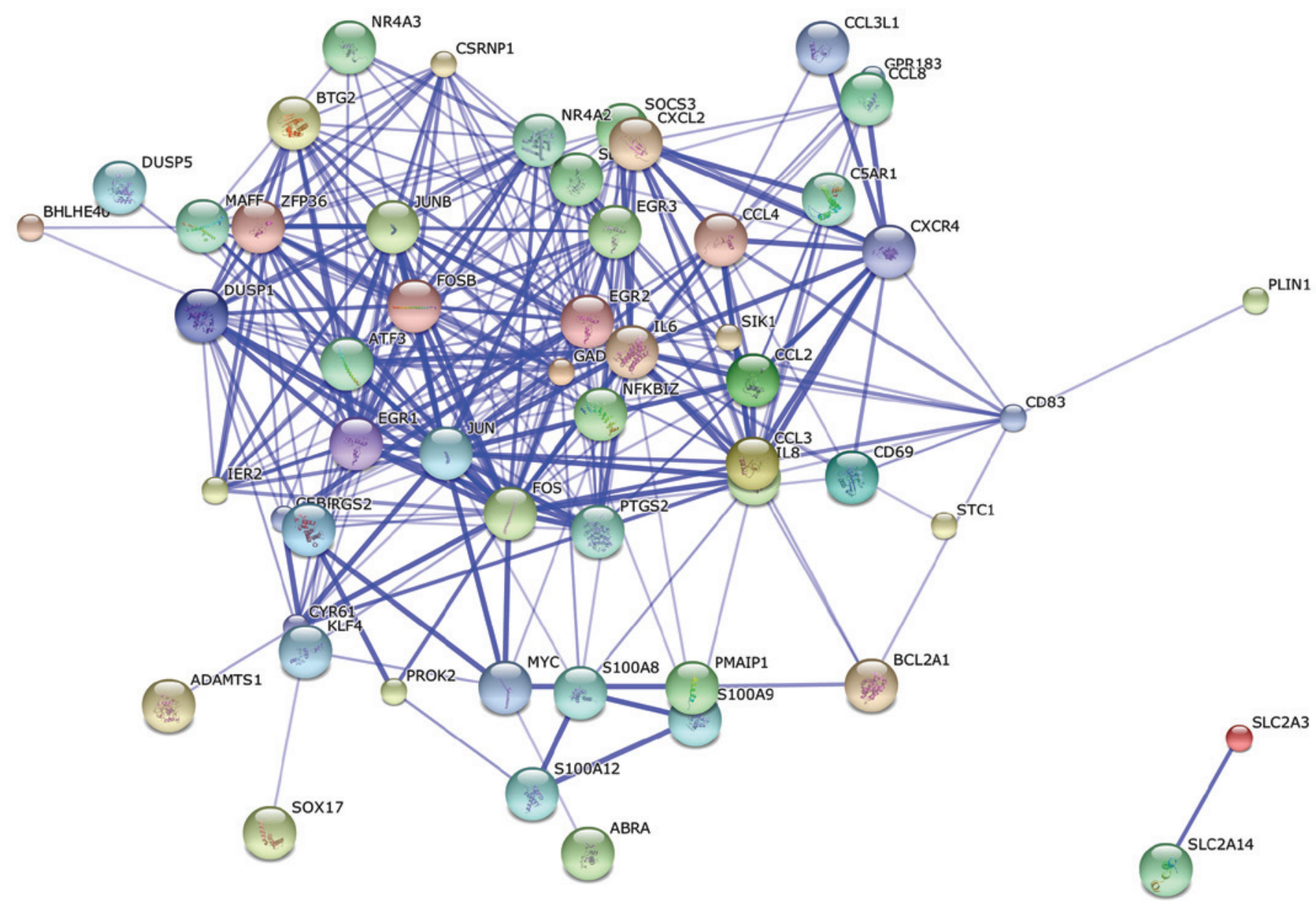

Figure 3. Protein-protein interaction networks of differently expressed genes. Thicknesses of edges represent the credibility of protein-protein interaction.

miRNA prediction analysis. In total, 36 miRNAs $(\mathrm{FDR}<0.05)$ were screened by GSEA enrichment analysis (Table V). MIR-224, which was the most significant miRNA with an FDR of 0.013101, regulated 152 target genes and had a GTGACTT target site. MIR-7 was also screened as a significant miRNA (FDR, 0.13673); it regulated 163 target genes and had a
GTCTTCC target site. Moreover, CAGGTCC was the target site of MIR-492 (FDR, 0.15391), which regulated 59 target genes. There were also several miRNAs that showed combined actions on one target site, such as MIR-34A, MIR-34C and MIR-449 (FDR, 0.017363), all of which acted on CACTGCC target sites and regulated a total of 273 target genes. 
Table IV. Top 10 nodes in the protein-protein interaction network.

\begin{tabular}{|c|c|c|c|c|c|c|c|}
\hline Node & Degree & $\log 2 \mathrm{FC}$ & P-value & Node & Degree & $\log 2 \mathrm{FC}$ & P-value \\
\hline FOS & 33 & 3.855915 & 0.000293 & JUNB & 23 & 1.970841 & $8.05 \mathrm{E}-05$ \\
\hline JUN & 25 & 1.174318 & 0.00015 & PTGS2 & 19 & 1.582406 & 0.000342 \\
\hline ATF3 & 25 & 1.655723 & 1.62E-05 & EGR1 & 18 & 2.609822 & $3.79 \mathrm{E}-06$ \\
\hline IL6 & 24 & 1.942007 & 0.000494 & EGR2 & 18 & 3.149878 & $9.33 \mathrm{E}-06$ \\
\hline FOSB & 23 & 2.974505 & 0.000304 & NR4A2 & 18 & 3.015238 & $3.16 \mathrm{E}-05$ \\
\hline
\end{tabular}

FC, fold change.

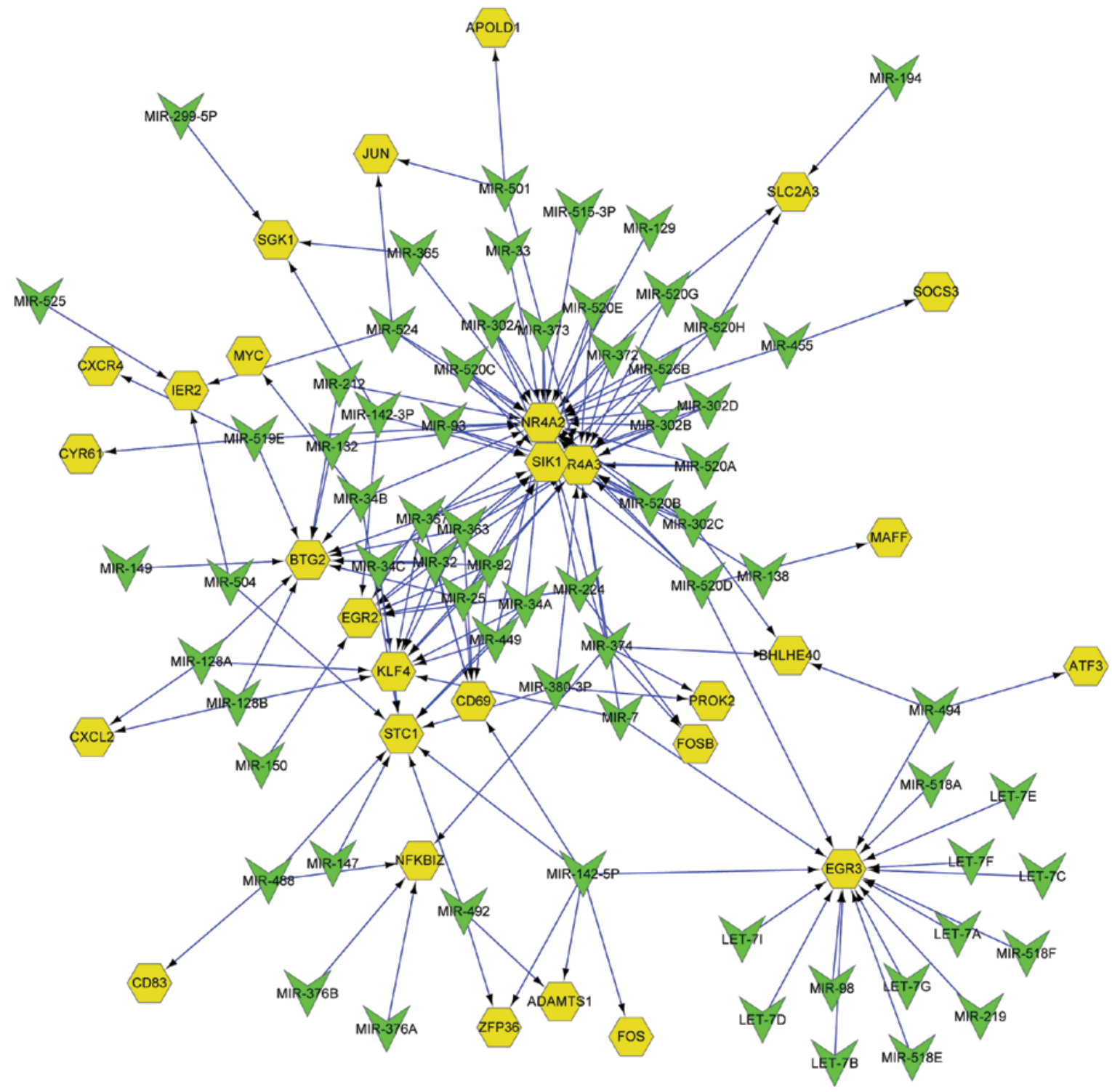

Figure 4. miRNA regulatory networks. Green nodes represent miRNAs, yellow nodes represent DEGs, blue edges represent regulatory relationships between miRNAs and DEGs, and black arrows represent the regulatory direction. miRNA, microRNA; DEG, differentially expressed gene.

miRNA regulatory network. An miRNA regulatory network was constructed from the screened miRNAs and the DEGs that they regulated. There were 176 edges and 97 nodes in the network, and the nodes consisted of 29 DEGs and 68 miRNAs (Fig. 4). All of the DEGs were regulated by one or several
miRNAs. For instance, EGR2 was regulated by 8 miRNAs, namely MIR-150, MIR-142-3P, MIR-367, MIR-363, MIR-32, MIR-92, MIR-25 and MIR-224, whereas ATF3 was only regulated by MIR-494. Similarly, each miRNA regulated one or more DEGs. MIR-142-5P regulated 6 DEGs simultaneously, 
Table V. Significantly enriched miRNAs.

\begin{tabular}{|c|c|c|c|c|c|}
\hline $\mathrm{Name}^{\mathrm{a}}$ & Size $^{b}$ & ES & NES & NOM P-value & FDR q-value \\
\hline GTGACTT, MIR-224 & 152 & 0.562058 & 1.822214 & 0 & 0.013101 \\
\hline GTCTTCC, MIR-7 & 163 & 0.437548 & 1.825578 & 0 & 0.013673 \\
\hline CAGGTCC, MIR-492 & 59 & 0.559375 & 1.757292 & 0 & 0.015391 \\
\hline AGCGCTT, MIR-518F, MIR-518E, MIR-518A & 18 & 0.632514 & 1.786852 & 0.019417 & 0.015701 \\
\hline CTGTTAC, MIR-194 & 102 & 0.514465 & 1.748545 & 0 & 0.015956 \\
\hline ATTACAT, MIR-380-3P & 100 & 0.568861 & 1.741315 & 0 & 0.01598 \\
\hline CAGGGTC, MIR-504 & 83 & 0.447092 & 1.763647 & 0 & 0.016012 \\
\hline CACTGCC, MIR-34A, MIR-34C, MIR-449 & 273 & 0.327004 & 1.722042 & 0 & 0.017363 \\
\hline GTAAACC, MIR-299-5P & 51 & 0.518946 & 1.692057 & 0 & 0.018053 \\
\hline ACACTAC, MIR-142-3P & 127 & 0.5551 & 1.722499 & 0 & 0.018404 \\
\hline TCTATGA, MIR-376A, MIR-376B & 81 & 0.461825 & 1.694669 & 0.007561 & 0.018496 \\
\hline TTGGGAG, MIR-150 & 89 & 0.463794 & 1.681071 & 0 & 0.019215 \\
\hline GGCACAT, MIR-455 & 57 & 0.545086 & 1.868148 & 0 & 0.019345 \\
\hline GCGCCTT, MIR-525, MIR-524 & 15 & 0.735336 & 1.694803 & 0.020121 & 0.019371 \\
\hline ACTGCCT, MIR-34B & 212 & 0.400143 & 1.70374 & 0 & 0.019561 \\
\hline GGCACTT, MIR-519E & 120 & 0.49198 & 1.675179 & 0 & 0.020529 \\
\hline AGGCACT, MIR-515-3P & 84 & 0.428035 & 1.666043 & 0 & 0.021838 \\
\hline GACTGTT, MIR-212, MIR-132 & 154 & 0.457287 & 1.655331 & 0 & 0.022945 \\
\hline TATTATA, MIR-374 & 282 & 0.49871 & 1.650721 & 0 & 0.023073 \\
\hline CACCAGC, MIR-138 & 217 & 0.31838 & 1.645393 & 0 & 0.024967 \\
\hline $\begin{array}{l}\text { CTACCTC, LET-7A, LET-7B, LET-7C, LET-7D, } \\
\text { LET-7E, LET-7F, MIR-98, LET-7G, LET-7I }\end{array}$ & 375 & 0.358576 & 1.62671 & 0 & 0.03133 \\
\hline GAGCCAG, MIR-149 & 143 & 0.30404 & 1.62081 & 0 & 0.031399 \\
\hline CCACACA, MIR-147 & 59 & 0.485473 & 1.621963 & 0 & 0.032141 \\
\hline ACTTTAT, MIR-142-5P & 283 & 0.475001 & 1.606214 & 0 & 0.034741 \\
\hline AAAGGAT, MIR-501 & 125 & 0.459692 & 1.600922 & 0 & 0.035487 \\
\hline $\begin{array}{l}\text { AGCACTT, MIR-93, MIR-302A, MIR-302B, } \\
\text { MIR-302C, MIR-302D, MIR-372, MIR-373, } \\
\text { MIR-520E, MIR-520A, MIR-526B, MIR-520B, } \\
\text { MIR-520C, MIR-520D }\end{array}$ & 330 & 0.465894 & 1.606906 & 0.005814 & 0.035811 \\
\hline CACTTTG, MIR-520G, MIR-520H & 230 & 0.487052 & 1.602 & 0.003876 & 0.036012 \\
\hline CAATGCA, MIR-33 & 91 & 0.479179 & 1.607379 & 0 & 0.036792 \\
\hline $\begin{array}{l}\text { GTGCAAT, MIR-25, MIR-32, MIR-92, } \\
\text { MIR-363, MIR-367 }\end{array}$ & 304 & 0.440998 & 1.581268 & 0 & 0.042634 \\
\hline ATGTTTC, MIR-494 & 155 & 0.455721 & 1.578166 & 0.015717 & 0.043117 \\
\hline GCAAAAA, MIR-129 & 182 & 0.431128 & 1.577369 & 0.031558 & 0.043279 \\
\hline GACAATC, MIR-219 & 138 & 0.403393 & 1.574232 & 0 & 0.0444 \\
\hline CTAGGAA, MIR-384 & 64 & 0.439962 & 1.566436 & 0 & 0.044528 \\
\hline CACTGTG, MIR-128A, MIR-128B & 327 & 0.344783 & 1.567878 & 0.005941 & 0.045063 \\
\hline TATCTGG, MIR-488 & 60 & 0.520626 & 1.568708 & 0 & 0.045787 \\
\hline GGGCATT, MIR-365 & 107 & 0.509769 & 1.558457 & 0.003883 & 0.04858 \\
\hline
\end{tabular}

anitial 7 bases are the target sites of the miRNA; ${ }^{b}$ size is the number of regulatory target genes. miRNA, microRNA; ES, enrichment score; NES, normalized enrichment score; NOM, normalized; FDR, false discovery rate (adjusted P-value).

namely CD69 molecule (CD69), ZFP36 ring finger protein (ZFP36), ADAM metallopeptidase with thrombospondin type 1 motif 1 (ADAMTS1), FOS, stanniocalcin 1 and early growth response 3 (EGR3), whereas MIR-194 only had a regulatory effect on solute carrier family 2 (facilitated glucose transporter), member 3 (SLC2A3). 


\section{Discussion}

OPCAB is a type of surgery that improves blood flow to heart. In this study, GSE12486 was downloaded from the GEO to investigate the effect of this surgery on myocardial tissue on the basis of molecular mechanisms. A total of 64 DEGs, including 63 upregulated and 1 downregulated DEGs were screened. PLIN1 was the unique downregulated DEG in this study, which has been confirmed to be associated with anterolateral myocardial infarction through various metabolic pathways and axon guidance $(23,24)$.

In addition, the present study also identified that the DEGs were mainly enriched in GO terms associated with chemokine activity, transcription factor activity and transcription regulator activity, and several KEGG pathways, such as chemokine signaling pathway, cytokine-cytokine receptor interaction, and Toll-like receptor signaling pathway. The FOS gene was enriched in various GO terms such as inflammatory response, defense response and transcription regulator activity. Wu et al have shown that FOS is an important target of immune inflammation responses in dendritic cells (25). The OPCAB surgery has been confirmed to activate an inflammatory response, specific hemostatic responses and immune mechanisms (26). Thus, the FOS gene may be involved in the changes that occur following OPCAB grafting via an immune mechanism. In addition, FOS, IL6 and JUN were the enriched genes in the Toll-like receptor and MAPK signaling pathways. The MAPK signaling pathway can be regulated by the activation of extracellular signal-regulated kinase (ERK)1 and 2, which can translocate to the nucleus and activate transcription factors, such as c-FOS (27). Inhibition of the activation of MAPK may attenuate warm ischemia-reperfusion injury (28), and MAPK inhibition is widely used to improve cardiac function after rewarming, cold storage and reperfusion (29). IL6 is an independent predictor for left ventricular systolic function in patients following OPCAB surgery (30). Furthermore, the ratio IL10/IL6 can be used as an inflammatory biomarker in OPCAB patients (31). In the present study, IL6 and IL8 were enriched in inflammation-related terms, such as chemotaxis, inflammatory response, defense response and response to wounding.

Moreover, a PPI network was constructed, and 10 hub nodes including FOS, JUN, ATF3, IL6, FOSB and JUNB were screened. Among them, FOS and JUN were the most important nodes with degrees of 33 and 25, respectively. A previous study has shown a direct association of signal transducer and activator of transcription 3 with c-FOS and c-JUN in response to IL6 (32). The Fos family, including c-FOS, FOSB and FOSB2, are able to form dimers with JUN proteins to regulate target genes (29). Furthermore, ATF3 has been confirmed to inhibit IL6 transcription by altering the structure of chromatin, and then restrict access to transcription factors (33). In the present study, FOS, JUN, IL6 and ATF3 were also screened and enriched in transcription factor activity. A correlation between FOS and EGR2 was also shown. EGR2, which is a human zinc finger-encoding gene, is induced with c-Fos-like kinetics by various mitogens in cells (34).

A total of 36 miRNAs were screened including MIR-494, MIR-501, MIR-524 and MIR-142-3P. Furthermore, an miRNA regulatory network was constructed based on the DEGs and screened miRNAs. It was found that ATF3 was only regulated by MIR-494. Similarly, Lan et al found that following reperfusion, the expression of ATF3 was inhibited by upregulated miRNA-494, and also associated with inflammation and adhesion (35). In addition, there was mutual regulated relationship between EGR2 and MIR-142-3P: ERG2 was encoded by the genes repressed by MIR-142-3P, while ERG2 associated with nerve growth factor-induced gene-A (NGFI-A) binding protein 2 binds to the pre-MIR-142-3p promoter to regulate its expression negatively (36).

In conclusion, the identified DEGs, such as FOS, IL6, EGR2 and JUN, might participate in biological processes including immune inflammation responses, the Toll-like receptor signaling pathway and the defense response of patients following OPCAB surgery. Moreover, the associations between DEGs and miRNA were screened. ATF3 was regulated by MIR-494, and JUN was regulated by MIR-501 and MIR494. However, these results require confirmation by experimental study.

\section{Acknowledgements}

The authors express warm thanks to Fenghe (Shanghai) Information Technology Co., Ltd., whose ideas and help provided a valuable added dimension to the study.

\section{References}

1. Worthley SG, Osende JI, Helft G, Badimon JJ and Fuster V: Coronary artery disease: Pathogenesis and acute coronary syndromes. Mt Sinai J Med 68: 167-181, 2001.

2. Nalysnyk L, Fahrbach K, Reynolds MW, Zhao SZ and Ross S: Adverse events in coronary artery bypass graft (CABG) trials: A systematic review and analysis. Heart 89: 767-772, 2003.

3. Kim NY, Shim JK, Bang SO, Sim JS, Song JW and Kwak YL: Effects of ulinastatin on coagulation in high-risk patients undergoing off-pump coronary artery bypass graft surgery. Korean J Anesthesiol 64: 105-111, 2013.

4. Arab S, Konstantinov IE, Boscarino C, Cukerman E, Mori A, Li J, Liu PP, Redington AN and Coles JG: Early gene expression profiles during intraoperative myocardial ischemia-reperfusion in cardiac surgery. J Thorac Cardiovasc Surg 134: 74-81.e2, 2007.

5. Gabrielsen A, Lawler PR, Yongzhong W, Steinbrüchel D, Blagoja D, Paulsson-Berne G, Kastrup J and Hansson GK: Gene expression signals involved in ischemic injury, extracellular matrix composition and fibrosis defined by global mRNA profiling of the human left ventricular myocardium. J Mol Cell Cardiol 42: 870-883, 2007.

6. Konstantinov IE, Coles JG, Boscarino C, Takahashi M, Goncalves J, Ritter J and Van Arsdell GS: Gene expression profiles in children undergoing cardiac surgery for right heart obstructive lesions. J Thorac Cardiovasc Surg 127: 746-754, 2004.

7. Chandrasekar B, Mitchell DH, Colston JT and Freeman GL: Regulation of CCAAT/Enhancer binding protein, interleukin-6, interleukin-6 receptor, and gp130 expression during myocardial ischemia/reperfusion. Circulation 99: 427-433, 1999.

8. Tomic V, Russwurm S, Möller E, Claus RA, Blaess M, Brunkhorst F, Bruegel M, Bode K, Bloos F, Wippermann J, et al: Transcriptomic and proteomic patterns of systemic inflammation in on-pump and off-pump coronary artery bypass grafting. Circulation 112: 2912-2920, 2005.

9. Ghorbel MT, Cherif M, Mokhtari A, Bruno VD, Caputo M and Angelini GD: Off-pump coronary artery bypass surgery is associated with fewer gene expression changes in the human myocardium in comparison with on-pump surgery. Physiol Genomics 42: 67-75, 2010.

10. Lan YF, Chen HH, Lai PF, Cheng CF, Huang YT, Lee YC, Chen TW and Lin H: MicroRNA-494 reduces ATF3 expression and promotes AKI. J Am Soc Nephrol 23: 2012-2023, 2012. 
11. Wang X, Zhang X, Ren XP, Chen J, Liu H, Yang J, Medvedovic M, Hu Z and Fan GC: MicroRNA-494 targeting both proapoptotic and antiapoptotic proteins protects against ischemia/reperfusion-induced cardiac injury. Circulation 122: 1308-1318, 2010.

12. Wang Z, Shao J, Zhou Q, Liu J, Zhu Y, Yang J and Wei M: The $-251 \mathrm{~A}>\mathrm{T}$ polymorphism of interleukin-8 is associated with longer mechanical ventilation and hospital staying after coronary surgery. Cytokine 50: 268-272, 2010.

13. Nathan N, Preux PM, Feiss P and Denizot Y: Plasma interleukin-4, interleukin-10, and interleukin-13 concentrations and complications after coronary artery bypass graft surgery. J Cardiothorac Vasc Anesth 14: 156-160, 2000.

14. Fromes Y, Daghildjian K, Caumartin L, Fischer M, Rouquette I, Deleuze P and BicalOM: A comparison of low vs conventional-dose heparin for minimal cardiopulmonary bypass in coronary artery bypass grafting surgery. Anaesthesia 66: 488-492, 2011.

15. Gautier L, Cope L, Bolstad BM and Irizarry RA: Affy-analysis of Affymetrix GeneChip data at the probe level. Bioinformatics 20 : 307-315, 2004

16. Diboun I, Wernisch L, Orengo CA and Koltzenburg M: Microarray analysis after RNA amplification can detect pronounced differences in gene expression using limma. BMC Genomics 7: $252,2006$.

17. Sartor MA, Tomlinson CR, Wesselkamper SC, Sivaganesan S Leikauf GD and Medvedovic M: Intensity-based hierarchical Bayes method improves testing for differentially expressed genes in microarray experiments. BMC Bioinformatics 7: 538, 2006.

18. Jolliffe I: Principal component analysis. In: Encyclopedia of Statistics in Behavioral Science. Everitt BS and Howell D (eds) John Wiley \& Sons, New York, NY, 2005.

19. Huang da W, Sherman BT and Lempicki RA: Systematic and integrative analysis of large gene lists using DAVID bioinformatics resources. Nat Protoc 4: 44-57, 2009.

20. Franceschini A, Szklarczyk D, Frankild S, Kuhn M, Simonovic M, Roth A, Lin J, Minguez P, Bork P, von Mering C and Jensen LJ: STRING v9.1: Protein-protein interaction networks, with increased coverage and integration. Nucleic Acids Res 41: D808-D815, 2013

21. Subramanian A, Tamayo P, Mootha VK, Mukherjee S, Ebert BL, Gillette MA, Paulovich A, Pomeroy SL, Golub TR, Lander ES and Mesirov JP: Gene set enrichment analysis: A knowledge-based approach for interpreting genome-wide expression profiles. Proc Natl Acad Sci USA 102: 15545-15550, 2005.

22. Shannon P, Markiel A, Ozier O, Baliga NS, Wang JT, Ramage D, Amin N, Schwikowski B and Ideker T: Cytoscape: A software environment for integrated models of biomolecular interaction networks. Genome Res 13: 2498-2504, 2003.

23. Jaffer I, Riederer M, Shah P, Peters P, Quehenberger F, Wood A, Scharnagl H, März W, Kostner KM and Kostner GM: Expression of fat mobilizing genes in human epicardial adipose tissue. Atherosclerosis 220: 122-127, 2012.
24. Lei P, Baysa A, Nebb HI, Valen G, Skomedal T, Osnes JB, Yang $Z$ and Haugen F: Activation of Liver X receptors in the heart leads to accumulation of intracellular lipids and attenuation of ischemia-reperfusion injury. Basic Res Cardiol 108: $323,2013$.

25. Wu C, Gong Y, Yuan J, Zhang W, Zhao G, Li H, Sun A, Kai Hu Zou Y and Ge J: microRNA-181a represses ox-LDL-stimulated inflammatory response in dendritic cell by targeting c-Fos. J Lipid Res 53: 2355-2363, 2012.

26. Levy JH and Tanaka KA: Inflammatory response to cardiopulmonary bypass. Ann Thorac Surg 75 (Suppl): S715-S720, 2003.

27. Junttila MR, Li SP and Westermarck J: Phosphatase-mediated crosstalk between MAPK signaling pathways in the regulation of cell survival. FASEB J 22: 954-965, 2008.

28. Kobayashi M, Takeyoshi I, Yoshinari D, Matsumoto K and Morishita Y: P38 mitogen-activated protein kinase inhibition attenuates ischemia-reperfusion injury of the rat liver. Surgery 131: 344-349, 2002.

29. Vassalli G, Milano G and Moccetti T: Role of mitogen-activated protein kinases in myocardial ischemia-reperfusion injury during heart transplantation. J Transplant 2012: 928954, 2012.

30. Mathew GM, Mathew DC, Lo SC, Alexios GM, Yang JC, Sashikumar JM, Shaikh TM and Huang CC: Synergistic collaboration of gut symbionts in Odontotermes formosanus for lignocellulosic degradation and bio-hydrogen production. Bioresour Technol 145: 337-344, 2013.

31. Mazandarani M, Mojtahedzadeh M, Yousefshahi F and Hamishehkar H: Effect of pre-treatment with hypertonic saline on IL-10/IL-6 ratio in CABG patient. Res Pharm Sci 7: S886, 2012.

32. Schuringa JJ, Timmer H, Luttickhuizen D, Vellenga E and Kruijer W: c-Jun and c-Fos cooperate with STAT3 in IL-6-induced transactivation of the IL- 6 response element (IRE). Cytokine 14: 78-87, 2001.

33. Gilchrist M, Thorsson V, Li B, Rust AG, Korb M, Roach JC, Kennedy K, Hai T, Bolouri H and Aderem A: Systems biology approaches identify ATF3 as a negative regulator of Toll-like receptor 4. Nature 441: 173-178, 2006

34. Rangnekar VM, Aplin AC and Sukhatme VP: The serum and TPA responsive promoter and intron-exon structure of EGR2, a human early growth response gene encoding a zinc finger protein. Nucleic Acids Res 18: 2749-2757, 1990.

35. Lan HY and Chung AC: TGF- $\beta /$ Smad signaling in kidney disease. Semin Nephrol 32: 236-243, 2012.

36. Lagrange B, Martin RZ, Droin N, Aucagne R, Paggetti J, Largeot A, Itzykson R, Solary E, Delva L and Bastie JN: A role for miR-142-3p in colony-stimulating factor 1-induced monocyte differentiation into macrophages. Biochim Biophys Acta 1833: 1936-1946, 2013 